\begin{tabular}{ccc}
\hline & International Journal of Engineering \&Technology, $7(3.12)(2018) 1317-1321$ \\
SPC & International Journal of Engineering \& Technology \\
Website: $w$ ww.sciencepubco.com/index.php/IJET & Research paper \\
\hline
\end{tabular}

\title{
Forest Fire Prevention Using WSN Assisted IOT
}

\author{
Vrince Vimal ${ }^{*}$, Madhav Ji Nigam ${ }^{2}$ \\ ${ }^{1,2}$ Electronics and Communication Engineering, Indian Institute of Technology, Roorkee, Uttarakhand, India \\ *Corresponding Author Email: ${ }^{1}$ vrince.vimal@gmail.com
}

\begin{abstract}
Internet of Things is the mainstay of the new era since its application becomes the future of day-to-day life. This work targets the IoT network assisted by WSN to prevent forest fire. We propose two-layer architecture of sensor network assisted by IoT enabled UAVs. The data flows in the proposed architecture in bottom-up fashion i.e., data is sensed by the nodes, which are deployed in the forest area (and sense temperature continuously). This data is transmitted to upper layer consisting of UAVs, which take appropriate action (to sprinkle water to bring temperature down to prevent fire). All the UAVs are interconnected to each other as well as to base station. The sensor nodes are clustered using two-step clustering algorithm, which takes care of the isolated nodes. The scheme has been equated to another WSN assisted IoT clustering technique. The proposed scheme outperforms the existing in terms of congestion at the UAV stations, number of alive nodes and remaining energy of the network.
\end{abstract}

Keywords: Forest Fire; WSN; Clustering; IoT.

\section{Introduction}

Forest fire roots massive damage to natural resources, environment as well as to an human lives [1]. It can be defined as unchecked fire taking place due to various reasons in forests. Sometimes, it is so worst that it takes days for fire fighters to take control of situation. The forest fire at times, is so large that it takes long time for the fire fighters to gain control over the situation. This sometimes results in massive destruction of natural resources. Fig. 1 shows the major forest with worst fires till date. The aim of this work is to propose a protocol for prevention of fire before it alleviates to uncontrollable level, as well as inform fire fighters to mend the situation well in time. In order to reach this objective, we have designed a outline of wireless sensor network (WSN) assisted IoT, taking in account some important characteristics which cause forest fire.

WSN consist of hundreds of sensor nodes. Every node has a detecting ability, calculation capability, and communicate via wireless medium.
These sensors are battery driven i.e. have limited energy source. Generally, are deployed in ad-hoc fashion in remote areas, so it is practically impossible to replace batteries. So, energy plays a paramount role in deciding the performance metrics of WSN [2]. To use the energy competently nodes are grouped into many small groups called clusters (Fig. 2). This technique of combining sensors is called as clustering [3].

For every cluster, depending upon various protocols one node is selected for communication with base station (BS) and is known as cluster head $(\mathrm{CH}) . \mathrm{CH}$ collects the data from all the sensors in the cluster the aggregated it to BS after fixed duration of time. Other nodes in the cluster are known as cluster members. $\mathrm{CH}$ is scalable for many sensor nodes and reduces energy consumption to increase network life time.

Selection of cluster head is an important issue in designing clustering protocols, and is reported widely in literature [4]-[9].

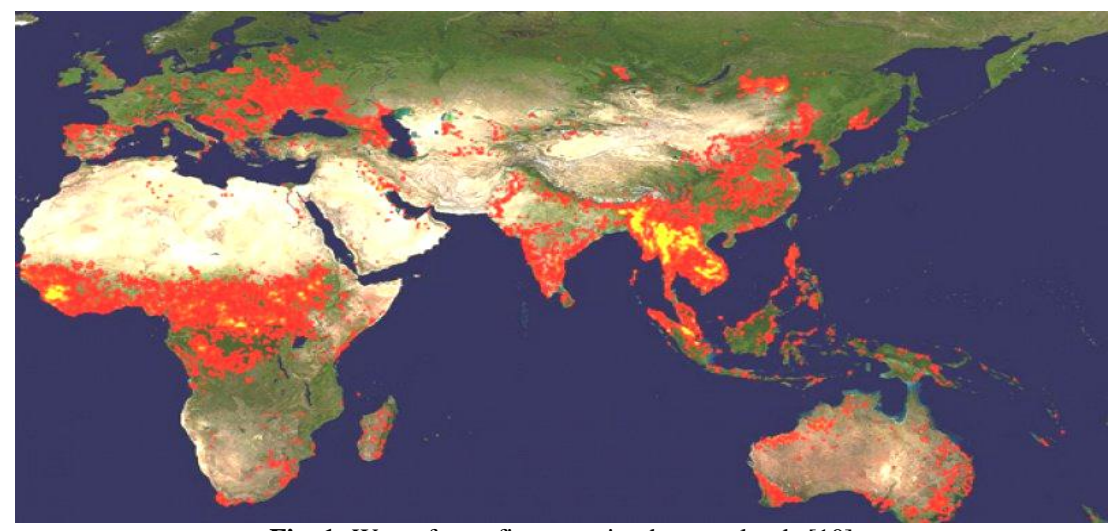

Fig. 1: Worst forest fires creating brown clouds [10] 


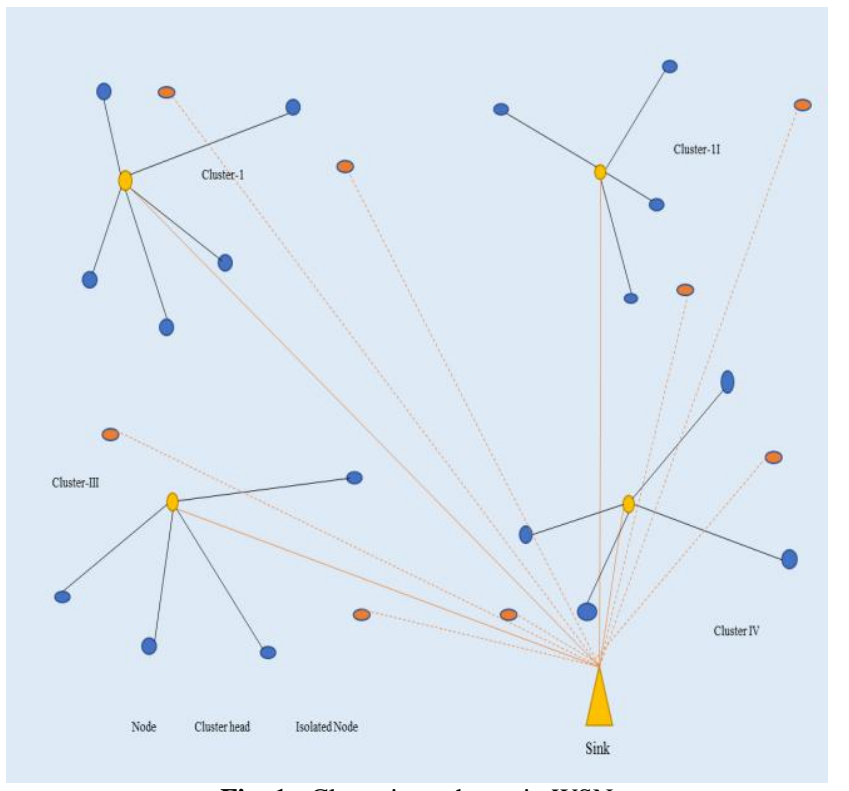

Fig. 1 : Clustering scheme in WSN

Wireless sensor networks are a classical IoT network, in which the nodes can sense, keep close watch of the network as well as transmit data to desired location. IOT can be defined as a vibrant omnipresent network, of things which are connected via wireless links. Can be individually recognized depending upon standard scalable and interoperable communication protocols [11]. Virtual and physical objects in the IoT have features and individualities that allow them to edge with information networks [12]. We can also refer IoT as physical objects connected globally, which can sense, process, and communicate with themselves and to external world due to embedded technology. These networks consist of very large heterogeneous nodes those are driven by limited power source, spectrum and processing capacity. These nodes have to mitigate with ever shooting data traffic needs parallel to maintai ing QoS requirements such as latency reliability and feedback loops. Initially IoT was dependent upon use of radio-frequency identification (RFID) technology, which is widely used in supply chain visibility, inventory tracking, logistics, library systems, pharmaceutical production and other industries. Though in last decade IoT has escalated up many phases and these technologies are accepted rapidly [13].

Here in this study we intend to prevent forest fire before it erupts using WSN assisted IoT. Some work has been reported in this direction. Zhang et al [14] proposed BEEM, where they evaluated the networks lifetime from QoS perspective. Though not much idea of how this BEEM protocol is used for detection and prevention of forest fire was deliberated.

In [15], Yu et al proposed real-time forest fire detection scheme by using WSN. Nodes sensed the temperature and relative humidity from forest and aggravate it to the $\mathrm{CH}$, where data was processed forming neural network. Sensed data was treated as input to neural network and produce an index which evaluates the probability of forest fire. The neural network takes the measured data as input to produce weather index, which measures the likelihood for the weather to cause a fire. Finally, manager node decides the fire danger probability based on received weather parameters and some other influencing factors. However, immediate prevention was not proposed in above protocol and it is well known that once the forest fire erupts, it become very difficult to control it.

In this paper, firstly, we propose two layer architecture, integrating WSN with Unmanned Arial Vehicle (UAV) for prevention of forest fire. We define a clustering algorithm for uniform coverage and uniform energy consumption of deployed sensor network. Secondly, we integrate WSN and UAV via IEEE 802.15.4 which defines the lower layers (PHY and MAC) of low-speed wireless local area networks (Low Rate, Low Power Wireless Personal
Area Networks - LR-WPAN) low latency communication protocol [16]. Whenever, a rise in temperature is detected by any of the sensor nodes, UAV will come at desired location and spray fire extinguisher chemical to combat the situation till the fire fighters are reached.

\section{Integrating WSN With IoT}

Integration of WSN with IoT is much more than a rumor, major technical firms are building their IoT scenario in tandem with WSN such as IBM's "A Smarter Planet", where sensors are considered to be basic building blocks in intelligent cities; Hp labs CeNSE which is deploying world wide web of sensors to create 'nervous system of planet' [17].

The problem is how sensor nodes would function when connecting WSN with the internet.it should either communicate directly or through the base station (BS). The 'thing' mentioned in Internet of things has to addressable and locatable physical thing via internet.

We assume that sensor nodes implement a compatible set of protocols such as 6LoWPAN, so that any internet host can have direct communication with the sensors \& vice versa.

\section{Previous Studies}

WSNs have been intensively incorporated for environment monitoring applications such as natural disaster, climate and animal monitoring also widely employs WSN. Some applications employ homogeneous networks, while other employ heterogeneous networks for more effective and selective sensing [18]. Many studies have reported composite concept where difference in nodes measurement is gathered and forwarded. Assembling of atomic actions or data detected by various different class of sensors is termed as composite sensing. WSN for fire detection has been reported widely [19]. Clustering is a most used scheme for data aggregation in WSNs. Many clustering algorithms are reported in existing literature, such as LEACH [3] and HEED [8]. In [20], authors used k-means and separate three classifiers, Feed Forward Neural Network (FFNN), Naive Bayes, and Decision Trees. All three classifiers were used to find type of data and to increase sensing and aggregation of data for a fire event. Data is divided into two separate class: event reporting fire event and disturbance. FFNN was found to perform better in terms of prediction accuracy than the rest two classifiers. Authors of Ref. [21] proposed a fuzzy logic based algorithm to select decision making nodes and detection nodes. Based on the data sensed by detection nodes and fuzzy rules, decision making nodes predict the real value of the environmental events and checks weather nodes is having failure or not. In Ref. [22], the authors proposed a Cluster-based Energy efficient Composite event detection (CEC) protocol. Any existing mechanism can be used for forming the clusters. All CHs form a backbone used to deliver reports to the sink. Each $\mathrm{CH}$ performs local data aggregation to detect events. When an event is detected, a report is sent to the sink along a backbone of CHs. Contrary to this fixed clustering, Ref.[23] Proposes an event-based clustering, where a cluster is initiated by and composed of nodes that detect events.

In WSNs the sink (or sinks) can be fixed or mobile. When the sink is mobile, the trajectory of the sink can be fixed, controlled (e.g., based on some parameters such as the residual energy) or random walk [24]. The Anchor-based Voronoi-scoping Routing Protocol [25] considers several sinks that move using a random path approach. Each sink chooses an anchor from its neighbors, based on the nodes' signal strength. The anchor sends a hello message to the sink's Voronoi scope neighbors, so that they know how to send the information to the sink. 


\section{Network Model}

Let the entire geographic region of forest be divided in to random zones depending upon feasibility. Let every zone area alienated in to ' $\mathrm{M}$ ' zones with area of $\mathrm{K} \times \mathrm{K}$ square units. Also presume that ' nod $_{i}$ ' be the number of nodes in ' $i$ 'th' zone of the network. Suppose ' $\rho_{s}$ ' is the density of nodes in zone ' $i$ ', of area $\mathrm{K} \times \mathrm{K}$ square units. (For the sake of simplicity and understanding, we assume network to be divided into 4 zones.

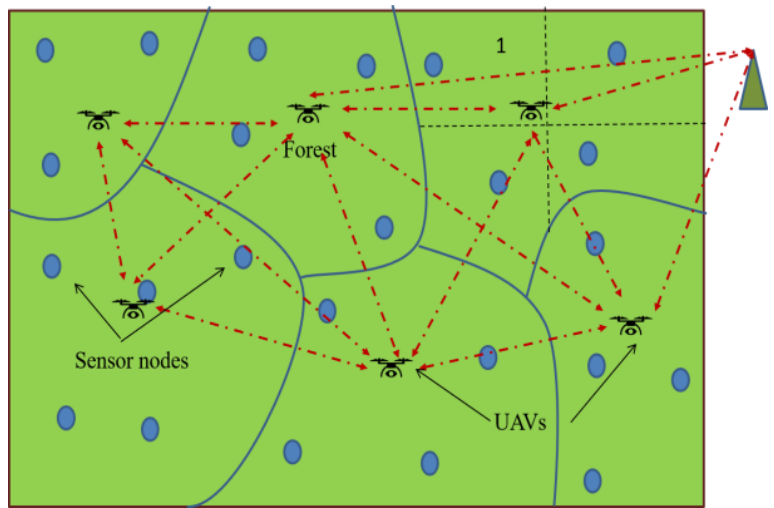

Fig. 3: Forest model showing initial deployment

Figure 3 shows the initial deployment of sensor nodes and forest divided in various zones depending upon geographic condition and each zone divided into ' $i$ ', zones. UAVs are initial placed at the centroid of each zone.

\subsection{Cluster Head Selection}

After initial deployment of sensor nodes in forest to ensure uniform coverage we make sure that cluster head is selected in every zone, or to provide uniformity to the cluster head election, each zone is required to have at least one cluster head. This would also ensure uniform energy consumption.

Now if one $\mathrm{CH}$ from every zone is selected, then this would mean out of $\mathrm{N}$ nodes, ' $\mathrm{k}$ ' number of nodes would act as cluster heads, such that $\mathrm{CH}=\left\{\mathrm{CH}_{1}, \mathrm{CH}_{2} \ldots \mathrm{CH}_{\mathrm{k}}\right\}$. Number of nodes that would be cluster members would be N-k or $\sum_{i=1}^{k}(m-1)$. For election of cluster heads, every node in set ' $\mathrm{M}_{\mathrm{k}}$ ' would compute fitness function based on zonal energy and zonal degree of connectivity. Suppose Fit ${ }^{\mathrm{m}, \mathrm{k}}=\left\{\mathrm{F}_{1}, \mathrm{~F}_{2} \ldots \mathrm{F}^{\mathrm{m}, \mathrm{k}}\right\}$ be the fitness set for each node ' $\mathrm{m}$ ' in $\mathrm{k}^{\text {th }}$ zone.

For any node, $\mathrm{n} \in \mathrm{CH}$, such that $\sum n=k$. All the nodes with

highest fitness function would be cluster head. In proposed scheme the residual energy and zonal average residual energy $\left(Z_{\text {Eav }}\right)$ of all sensors in each cluster along with the zonal average degree of connectivity $\left(Z_{D O C}\right)$ are considered to designate cluster heads.

Zonal average residual energy is the average energy remaining of nodes in particular zone and can be formulated as:

$$
Z_{D o c}=\sum_{i=1}^{M}(D o c(i)) / M
$$

On the other hand, Degree of connectivity for any node would be equal to number of connections in the communication range of a node. Finally, the node having the highest weight considering degree of connectivity and residual energy will be designated as cluster head.

$$
W_{d} \times\left(D O C(i) / Z_{D o c}\right)+W_{e} \times\left(E_{i} / Z_{E a v}\right) .
$$

For all nodes $\mathrm{n} \in \mathrm{CH}$, an ADV packet is sent in the range ' $\mathrm{R}$ ' to the nodes in the neighbor set. Nodes that would receive the ADV packet would join the cluster head to form the cluster. Such set of nodes would be, $\delta=\{\delta 1+\delta 2+\ldots .+\delta \sigma\}$, such that $1<\sigma<N-k$.

\subsubsection{Degree of Connectivity}

The proposed algorithm will begin with base station sending HELLO packets to every node in the network so that a node can know of its zone. The Hello packet would contain Id of zone and nodes lying within the zone. Every node can calculate distance from Received signal strength of the Hello packet as follows,

$d_{b u}=10^{\left[\left(P_{O}-F_{m}-\mathrm{P}_{\mathrm{r}}-10 \times n \times \log 10(f)+30 \times n-32.44\right) / 10 \times n\right]}$

Solving equation (3) for localization model, we get,

$$
\left\{\begin{array}{l}
X=\frac{x_{1}+x}{2} \\
Y=\frac{y_{1}+y}{2}
\end{array}\right.
$$

Once coordinates are found as in Equation (4), every node can compute its distance from every other node in the network using Euclidean distance formula. All those distance values for particular node which are less than communication range of a node, will act as its neighbors. The number of neighbors for a particular node would be its degree of connectivity in the network.

Clustering Isolated nodes

For prolonging the networks lifetime we propose further clustering of isolated nodes, which are in communication range of each other [2]. Nodes that do not receive any ADV message would belong to set $\varsigma=\left\{\varsigma_{1}, \varsigma_{2}, \ldots, \varsigma_{\varphi}\right\} \quad$ would be isolated nodes, such that $1<\varphi<\sigma \& \varphi+\sigma=N-k \operatorname{or} \varphi+\sigma=\sum_{i=1}^{k}(M-1)$. For all the nodes belonging to set $\varsigma$, if $D\left(\varsigma^{i, i+1}\right)<R$, then they must form clusters using latter clustering algorithm. Say, $\gamma$ represent the number of nodes that would form' $\lambda$ ' clusters out of ' $\varphi$ ' number of isolated nodes, such that $\varphi=\gamma+\lambda+\mu$. Where' ' $\mu$ ' is nodes which are still isolated after second step clustering. This step will ensure that, even if some of the nodes have been devoid of clusters formed initially, their clustering would reduce their energy consumption while interacting with the base station. Now in the final isolated nodes set $\mu=\left(\sum_{i=1}^{k}(M-1)\right)-\sigma-\gamma-\lambda$ number of nodes remain. These nodes would directly communication with base station. The objective function would be to minimize ' $\mu$ ':

$M I N \mu=\min \left(\left(\sum_{i=1}^{k}(M-1)\right)-\sigma-\gamma-\lambda\right)$

Energy consumed by the WSN would be :

$E=K \times E_{C H}+\lambda \times E_{C H I S O}+(\sigma+\gamma) \times E_{m e m}+\mu \times E_{i s o}$

Putting values to WSN energy equation: 


$$
\begin{aligned}
E= & \int_{i=1}^{\left(\sum_{i=1}^{k}(M-1)\right)-\sigma-\gamma-\lambda}\left(E_{\text {electronics }} \times L+E_{\text {amplifier }} \times D_{i}, B S^{2}\right)+ \\
& \int_{i=1}^{\gamma}\left(E_{\text {electronics }} \times L+E_{\text {amplifier }} \times D_{i}, \lambda^{2}\right)+\int_{i=1}^{\lambda}\left(E_{\text {agg }} \times L \times \gamma\right)+ \\
& \left(E_{\text {electronics }} \times L+E_{\text {amplifier }} \times D_{i}, B S^{2}\right)+ \\
& \int_{i=1}^{\sigma}\left(E_{\text {electronics }} \times L+E_{\text {amplifier }} \times D_{i}, \sigma^{2}\right)+\int_{i=1}^{k}\left(E_{\text {agg }} \times L \times \sigma\right)+ \\
& \left(E_{\text {electronics }} \times L+E_{\text {amplifier }} \times D_{i}, B S^{2}\right) .
\end{aligned}
$$

\section{Proposed Model}

Since the area of single zone of a forest tends to be large, thus the nodes deployed in that area tends to follow TSUC clustering technique to group the nodes in several clusters. Each node will continuously monitor the environment in the idle mode. The fact that the nodes remain in the idle mode for most of time tends to make this system more energy efficient. These sensor nodes need to turn on their radios for data transmission only if the value of temperature sensed by them exceeds certain threshold limit. The value of threshold limit of the temperature depends on the real-time conditions and will be set at less than the value at which grass or leaves catches fire. For example, if the temperature at which leaves can burn is 50 Celsius, then the threshold value can be set at 10 percent lower to the upper-limit value. That means as soon as the temperature hits 45 Celsius, the sensor nodes will forward the data to the IoT assisted UAVs, which can come in for sprinkling to reduce the temperature and thus preventing the disaster to the flora and fauna.

The main objective of the work is to reduce the latency of reporting the data from sensors to the UAVs. This is done by formulating a fitness function for the relay nodes. Each node will forward the data in multi-hop nature to the UAVs. Selection of relay node however, depends upon the fitness function of each node. We define fitness function as:

$$
F=\alpha \times D(R, U A V s)+\beta \times(\text { Remaining Energy })
$$

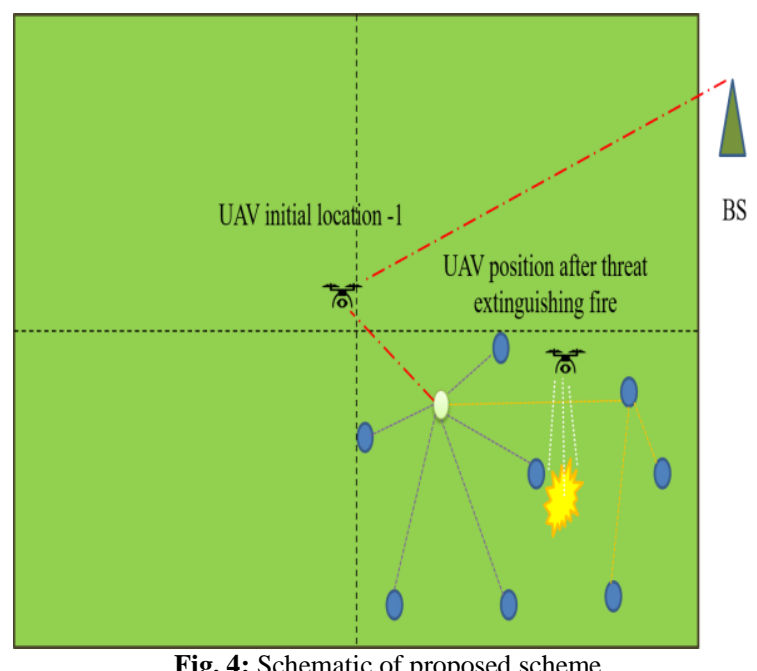

Fig. 4: Schematic of proposed scheme

Figure 4 shows the schematic of the proposed algorithm.

Where D (R, UAVs) is the distance between the relay node and UAV station.

At the second layer, all the UAV stations need to be positioned in the center for zone such that communication cost from the sensor to the UAV station is less. Each station houses transmitter and receivers, which can communicate to the nearest UAV station. This is mandatory for the fact that if UAVs in one of the station goes empty, UAVs from other station can join in. Finally, the UAVs station need to be connected to main station at outside of the forest or easily accessible human area

\section{Results}

We compared the TSUC IoT performance with ECBR IoT [26]. Comparisons were made on four parameters namely remaining energy of the network, number of alive nodes, through put, remaining energy of the isolated nodes and number of isolated nodes in the network

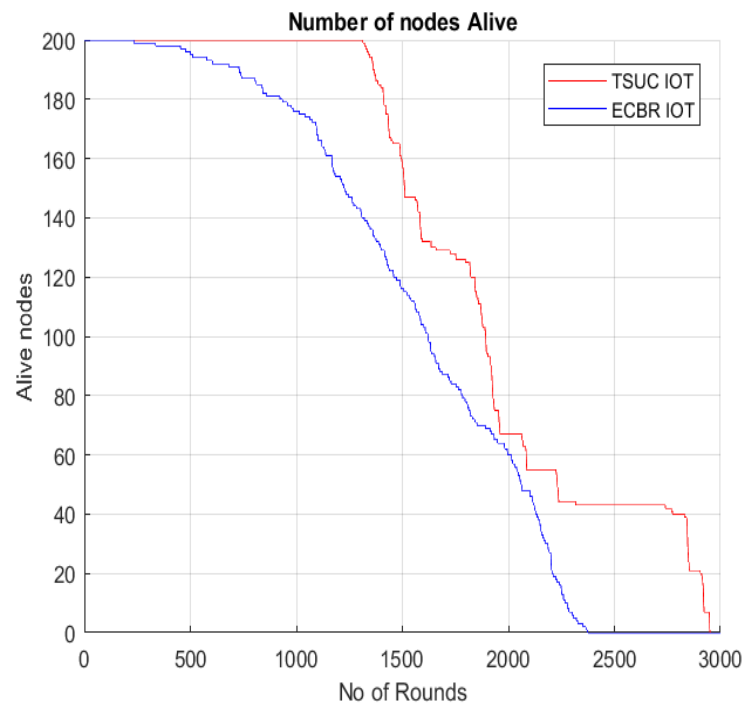

Fig. 5: Number of nodes alive

Energy consumed is lesser for proposed scheme than other ECBR IoT, this will also improve the lifetime of the network which is adequately proven from the graph of number of alive nodes in the network Figure 5. As is apparent from the graph of number of alive nodes that all the nodes are alive till approximately over 700 rounds, this is because of the fact that energy is consumed uniformly from the entire network as every zone has cluster head, unlike other schemes where nodes nearer to the sink tend to die early, network goes completely dead at around 2700 rounds for TSUC. ECBR however, performs slightly better than other available schemes in context with number of alive nodes and remaining energy of the network, this is because ECBR allows the isolated nodes to transfer the data to base station via cluster heads (those cluster heads to which these nodes had formed clusters in the previous rounds).

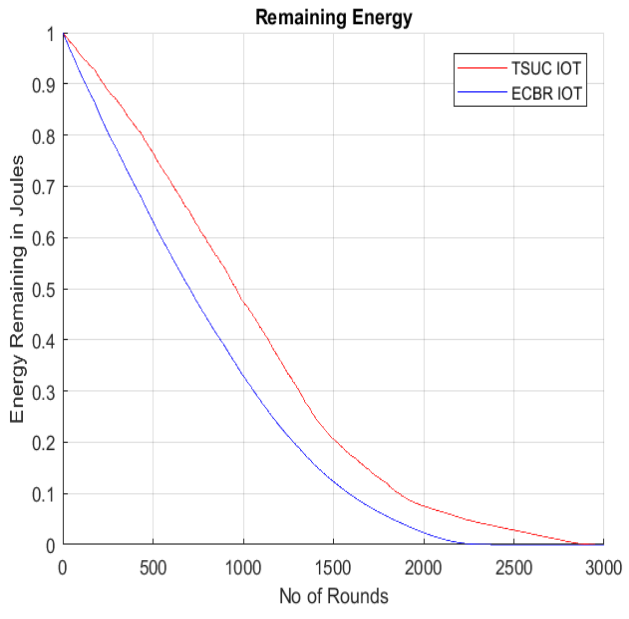

Fig. 6: Remaining energy 
Figure 6 shows that energy is uniformly drained from entire network and network is well alive after 2500 rounds as well.

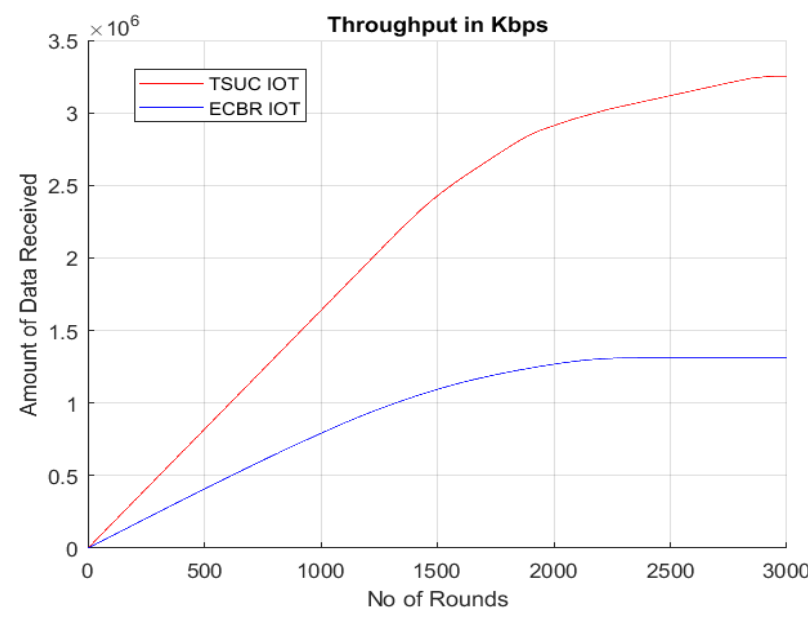

Fig. 7: Throughput

The throughput has been plotted as cumulative throughput for the successive rounds Figure 7. Each node sends a packet for 1024 bytes to its respective cluster head or to base station directly in case of isolated nodes. As number of alive nodes keep getting less, the value of throughput starts becoming constant in the network.

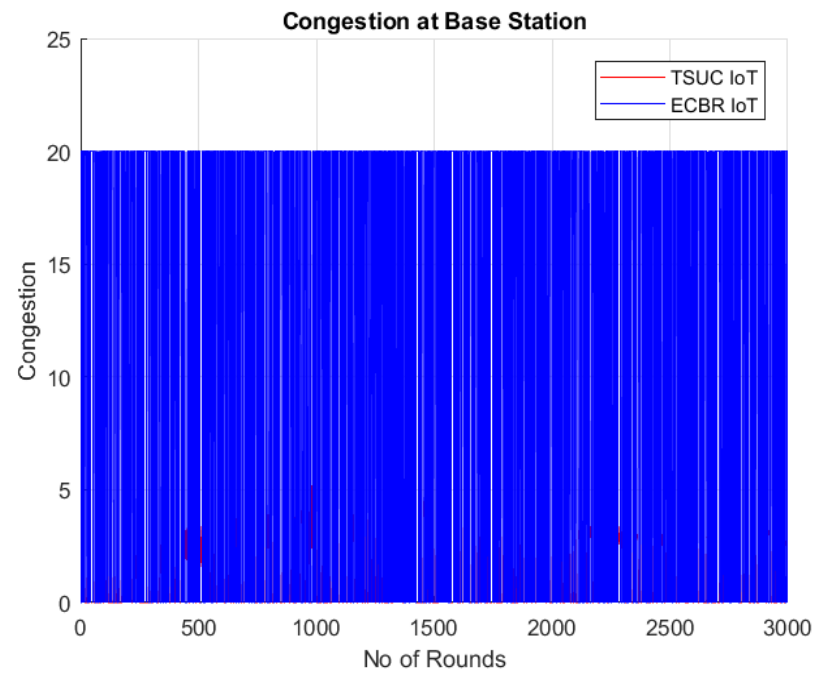

Fig. 8: Congestion in proposed scheme

\section{Conclusion}

In this paper, we proposed a forest fire modell, which includes a flexible and scalable platform to both deploy algorithms based on proposed clustering algorithm and sensing model for forest fire. Specifically, it is a fusion of WSN and IoT and a two-layer architecture for prevention of forest fire. It considers relevant characteristics that may raise temperature and uses a clustering algorithm for planning and prioritizing the extinction of the cause of fire. The results show that propose algorithm has improved sensing coverage and prolonged networks lifetime.

\section{References}

[1] S. Sharma, "Virtual and augmented reality: Applications in emergency fire evacuation for training and education"," 2016.

[2] V. Vimal and M. J. Nigam, "Ensuring uniform energy consumption in non- deterministic wireless sensor network to protract networks lifetime,” Int. J. Electron. Commun. Eng., vol. 11, no. 9, pp. 966-970, 2017.
[3] W. R. Heinzelman, A. Chandrakasan, and H. Balakrishnan, "Energyefficient communication protocol for wireless microsensor networks,' Proc. 33rd Annu. Hawaii Int. Conf. Syst. Sci., vol. 0, no. c, pp. 30053014, 2000.

[4] H. Wang, N. Agoulmine, M. Ma, and Y. Jin, "Network lifetime optimization in wireless sensor networks," IEEE J. Sel. Areas Commun., vol. 28, no. 7, pp. 1127-1137, 2010.

[5] S. Yi, J. Heo, Y. Cho, and J. Hong, "PEACH: Power-efficient and adaptive clustering hierarchy protocol for wireless sensor networks," Comput. Commun., vol. 30, no. 14-15, pp. 2842-2852, 2007.

[6] W. Ke, O. Yangrui, J. Hong, Z. Heli, and L. Xi, "Energy aware hierarchical cluster-based routing protocol for WSNs," J. China Univ. Posts Telecommun., vol. 23, no. 4, pp. 46-52, 2016.

[7] D. Kumar, T. C. Aseri, and R. B. Patel, "EEHC: Energy efficient heterogeneous clustered scheme for wireless sensor networks," Comput. Commun., vol. 32, no. 4, pp. 662-667, 2009.

[8] C. H. Lin and M. J. Tsai, "A Comment on "HEED: A Hybrid, energyefficient, distributed clustering approach for ad hoc sensor networks," IEEE Trans. Mob. Comput., vol. 5, no. 10, pp. 1471-1472, 2006.

[9] L. Zhao and Q. Liang, "Medium-contention based energy-efficient distributed clustering (MEDIC) for wireless sensor networks," Int. J. Distrib. Sens. Networks, vol. 3, no. 4, pp. 347-369, 2007.

[10] J. Vidal, "Forest Fires Feed 'Brown Cloud' of Pollution Choking Asia's Cities," Raw Story, 2014. [Online]. Available: https://www.rawstory.com/2014/03/indonesian-forest-fires-feed-browncloud-of-pollution-choking-asias-cities/. [Accessed: 22-Mar-2018].

A. Whitmore, A. Agarwal, and L. Da Xu, "The Internet of Things-A survey of topics and trends," Inf. Syst. Front., vol. 17, no. 2, pp. 261-274, 2015

[11] R. Jadhav and P. Rajalakshmi, "IoT enabled smart and secure power monitor,” TENSYMP 2017 - IEEE Int. Symp. Technol. Smart Cities, 2017

[12] Y. Sivakrishna et al., "Performance analysis of hybrid multiple radio IoT architecture for ubiquitous connectivity," IEEE World Forum Internet Things, WF-IoT 2015 - Proc., pp. 198-203, 2015.

[13] Y. Zhang, N. Cao, G. Chang, L. Zhou, X. Yu, and Y. Liu, "Wireless Sensor Routing Protocol Research Based on Forest Fire Protection,' IEEE Int. Conf. Comput. Sci. Eng. IEEE Int. Conf. Embed. Ubiquitous Comput., pp. 265-268, 2017.

[14] Liyang Yu, Neng Wang, and Xiaoqiao Meng, "Real-Time Forest Fire Detection With Wireless Sensor Networks," Proceedings. 2005 Int Conf. Wirel. Commun. Netw. Mob. Comput. 2005., vol. 2, pp. 1214 1217, 2005.

[15] L. F. Schrickte, C. Montez, R. De Oliveira, and A. R. Pinto, "Integration of wireless sensor networks to the internet of things using a 6LoWPAN gateway,” Brazilian Symp. Comput. Syst. Eng. SBESC, pp. 119-124, 2014.

[16] C.Alcarez, P. Najera, and R. Roman, "Wireless Sensor Networks and the Internet of Things: Do We Need a Complete Integration?," in International Workshop on the Security of the Internet of Things (SecIoT10), 2010, vol. 6, no. 11, p. 113

[17] R. Kumar, S. K. Panda, and D. C. Hoang, "Realisation of a clusterbased protocol using fuzzy C-means algorithm for wireless sensor networks," IET Wirel. Sens. Syst., vol. 3, no. 3, pp. 163-171, 2013.

[18] M. Bahrepour, N. Meratnia, P. Havinga, and P. S. Group, "Automatic Fire Detection : a Survey From," Structure, 2007.

[19] M. O. Oladimeji, M. G. Smiee, and S. D. Mieee, "A new approach for event detection using k-means clustering and neural networks," Int. Jt Conf. Neural Networks, pp. 1-5, 2015.

[20] S. Zhang, H. Chen, Q. Zhu, and J. Jia, "A Fuzzy-Decision Based Approach for Composite Event Detection in Wireless Sensor Networks,' Sci. World J., vol. 2014, pp. 1-20, 2014.

[21] I.Memon and T. Muntean, "Cluster-based Energy-efficient Composite Event Detection for Wireless Sensor Networks," no. c, pp. 241-247, 2012.

[22] C.A. Suescun and M. Cardei, "Event-based Clustering for Composite Event Detection in Wireless Sensors Networks," in IEEE 35th International Performance Computing and Communications Conference (IPCCC), 2016.

A. Waheed Khan, A. H. Abdullah, M. H. Anisi, and J. Iqbal Bangash, "A comprehensive study of data collection schemes using mobile sinks in wireless sensor networks," Sensors (Switzerland), vol. 14, no. 2, pp. 2510-2548, 2014.

[23] K.T. K. Tian, B. Z. B. Zhang, K. H. K. Huang, and J. M. J. Ma, "Data Gathering Protocols for Wireless Sensor Networks with Mobile Sinks," GLOBECOM 2010, 2010 IEEE Glob. Telecommun. Conf., 2010.

[24] J.Shen, A. Wang, C. Wang, P. C. K. Hung, and C. F. Lai, "An efficient centroid-based routing protocol for energy management in WSNassisted IoT,” IEEE Access, vol. 5, pp. 18469-18479, 2017. 